\title{
Quantum Limit for Laser Interferometric Gravitational-Wave Detectors from Optical Dissipation
}

\author{
Haixing Miao, ${ }^{1}$ Nicolas D. Smith, ${ }^{2}$ and Matthew Evans ${ }^{3}$ \\ ${ }^{1}$ School of Physics and Astronomy, and Institute for Gravitational Wave Astronomy, \\ University of Birmingham, Birmingham B15 2TT, United Kingdom \\ ${ }^{2}$ California Institute of Technology, Pasadena, California 91125, USA \\ ${ }^{3}$ Massachusetts Institute of Technology, Cambridge, Massachusetts 02139, USA
}

(Received 2 August 2018; revised manuscript received 1 January 2019; published 26 March 2019)

\begin{abstract}
We derive a quantum limit to the sensitivity of laser interferometric gravitational-wave detectors from optical-loss-induced dissipation, analogous to the sensitivity limit from the mechanical dissipation. It applies universally to different interferometer configurations and cannot be surpassed unless the optical properties of the interferometer are improved. This result provides an answer to the long-standing question of how far we can push the detector sensitivity given the state-of-the-art optics.
\end{abstract}

DOI: 10.1103/PhysRevX.9.011053

Subject Areas: Gravitation, Optics, Quantum Physics

\section{INTRODUCTION}

Advanced gravitational-wave (GW) detectors are longbaseline interferometers with suspended mirrors which act as test masses for probing spacetime dynamics. Quantum noise, arising from quantum fluctuations of the optical field, is one of the sources of noise that limits the sensitivity of such instruments. In particular, the phase fluctuation gives rise to the shot noise, while the amplitude fluctuation exerts a random force on the test masses and induces the quantum radiation pressure noise. These two types of quantum noise, when uncorrelated, lead to the standard quantum limit (SQL) [1,2] of which the power spectral density is

$$
S_{h h}^{\mathrm{SQL}}(\Omega)=\frac{8 \hbar}{M \Omega^{2} L^{2}},
$$

where $\Omega$ is the angular frequency of the GW signal, $M$ is the mass of the test-mass mirror, and $L$ is the interferometer arm length.

Despite its name, the SQL is not a true limit: It can be surpassed with a wide class of so-called quantumnondemolition (QND) schemes (cf. review articles [3-5]). These techniques usually involve modifications to the optical configuration of current-generation GW detectors by introducing extra optical filters. These filters can be a cascade of both passive Fabry-Perot cavities and active cavities that have external energy input [6-8]. For example,

Published by the American Physical Society under the terms of the Creative Commons Attribution 4.0 International license. Further distribution of this work must maintain attribution to the author(s) and the published article's title, journal citation, and DOI. together with a squeezed light source, a passive filter cavity can be used for producing the frequency-dependent squeezing [9-11]. The general scheme is illustrated in Fig. 1.

The quantum Cramér-Rao bound (QCRB) $[12,13]$ is a sensitivity limit that, unlike the SQL, is inviolable. In the context of GW detection with laser interferometers, the QCRB is also called the energetic or fundamental quantum limit $[14,15]$. The spectral representation of the QCRB is

$$
S_{h h}^{\mathrm{QCRB}}(\Omega)=\frac{\hbar^{2} c^{2}}{2 S_{P P}(\Omega) L^{2}}
$$

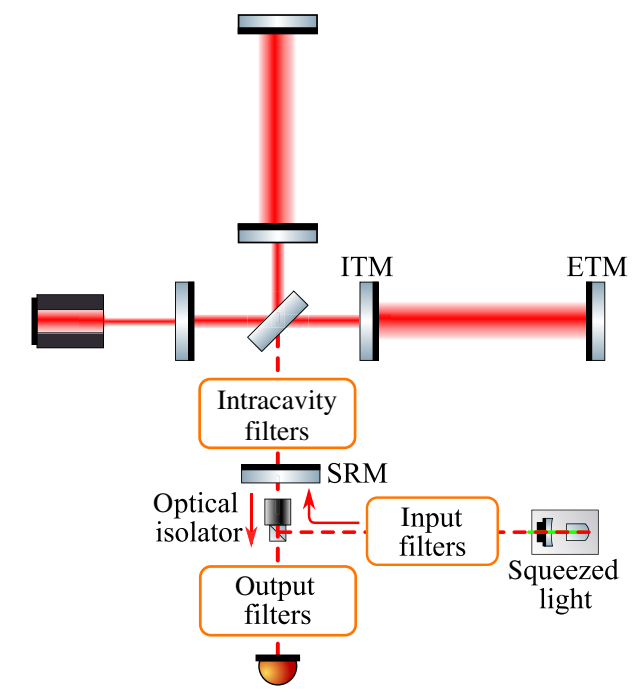

FIG. 1. Illustration of the general QND scheme with optical filters added to the typical configuration of advanced $\mathrm{GW}$ detectors-a dual-recycled Michelson interferometer. (ETM: end test mass; SRM: signal-recycling mirror.) 
in which $S_{P P}$ is the spectral density of the power fluctuations in the interferometer arms. As shown in Ref. [16], the QCRB can be approached in a lossless system with optimal frequency-dependent homodyne read-out, which can be realized with proper output filters [9]. This result has been generalized to laser interferometers with multiple carrier frequencies [17].

According to the QCRB, the sensitivity is ultimately bounded by the power or, equivalently, energy fluctuation inside the arm cavities. This can be intuitively understood from the fact that we want to measure the phase or timing difference between the two interferometer arms accurately, and a large uncertainty in the photon number or energy is needed, due to the number-phase or energy-time uncertainty relation. Note, however, that increasing $S_{P P}$ is only advantageous if a minimum uncertainty state, or at least nearly minimum uncertainty state, can be maintained. Minimum uncertainty states are, however, very delicate and easily destroyed by optical losses which lead to decoherence.

The power fluctuation $S_{P P}$ can be enhanced, and thus the QCRB can be reduced, with a variety of approaches. The most obvious approach is to increase the optical power in the interferometer, but this is limited by thermal lensing [18], alignment stability [19], and parametric instabilities $[20,21]$. A second approach is to introduce squeezed states of light generated by nonlinear optical processes [11] at the read-out port (cf. Fig. 1). In addition to squeezing the vacuum fluctuations that enter the interferometer, the radiation pressure coupling between the optical field and the test masses produces squeezing internally. This process, known as "ponderomotive squeezing" $[9,22]$, is actually the cause of the radiation pressure noise. We can turn it into a resource for enhancing the detector sensitivity by detuning the signal-recycling cavity (SRC) formed by input test mass (ITM) and SRM away from the perfect resonance. This is the so-called optical spring effect that was studied extensively by focusing on the dynamics of the test masses [23]. In Appendix A, we illustrate an equivalent picture mentioned in Ref. [16] from the perspective of the internal ponderomotive squeezing.

In principle, we can combine the above-mentioned approaches to increase $S_{P P}$ and make the QCRB vanishingly small, which implies an unbounded sensitivity. A clear example of this would be the injection of a very strongly squeezed state, which could increase $S_{P P}$ by more than an order of magnitude. In the presence of optical losses, a highly squeezed state will cease to be a minimum uncertainty state due to decoherence, and the QCRB in the lossless case will not be a relevant bound.

In this paper, we present a new general limit to GW detectors, which cannot be surpassed and is more constraining than the QCRB for realistic interferometer configurations with optical losses. As we will show, the optical losses lead to the following sensitivity limit, to the first order of the loss parameter $\epsilon$ :
$S_{h h}^{\epsilon}=\frac{\hbar c^{2}}{4 L^{2} \omega_{0} P}\left[\epsilon_{\mathrm{arm}}+\left(1+\frac{\Omega^{2}}{\gamma^{2}}\right) \frac{T_{\mathrm{itm}} \epsilon_{\mathrm{src}}}{4}+\alpha T_{\mathrm{src}} \epsilon_{\mathrm{ext}}\right]$.

Here, $P$ is the optical power inside each arm (assumed to be equal); $\omega_{0}$ is the laser frequency; $\epsilon_{\text {arm }}$ quantifies the internal loss of the arm cavity (e.g., $\epsilon_{\mathrm{arm}}=10^{-6}$ for $1 \mathrm{ppm}$ loss); $\epsilon_{\mathrm{src}}$ quantifies the optical loss inside the SRC, including additional intracavity filters if any; $\epsilon_{\mathrm{ext}}$ denotes the external loss, which includes the loss in the output chain and also the quantum inefficiency of the photodetection; $\gamma$ is the bandwidth of the arm cavity and is equal to $c T_{\mathrm{itm}} /(4 L)$, with $T_{\mathrm{itm}}$ being the power transmission of ITM; $\alpha$ is equal to 1 if we use the internal squeezing to maximize the power fluctuation and $\alpha$ is equal to $1 / 4$ if instead the internal squeezing is negligible, which has to do with the effect of internal squeezing on the signal response [24,25] [cf. Eq. (20) and also Appendix A]; $T_{\text {src }}$ is the effective transmissivity of SRC, which may be frequency dependent.

The arm-cavity loss sets a flat limit across different frequencies, as the additional vacuum fluctuation is directly mixed with the signal inside the arm. The SRC loss is suppressed by ITM transmission at low frequencies. However, due to a finite arm-cavity bandwidth, which reduces the signal response, this effect becomes important at high frequencies. The effect of the external loss depends on the transmission of SRC, which can be frequency dependent. For Advanced LIGO, $\epsilon_{\text {arm }}$ is of the order of $10^{-4}(100 \mathrm{ppm}), \epsilon_{\mathrm{src}}$ is around $10^{-3}$, which mainly comes from the beam splitter, and $\epsilon_{\mathrm{ext}}$ is around 0.1 (coming from the mode mismatch to the output mode cleaner and photodiode quantum inefficiency [26]). Given $T_{\mathrm{itm}}=$ 0.014 and $T_{\text {src }} \approx 0.14$ in the default broadband detection mode, we show the resulting sensitivity bound imposed by current loss values in Fig. 2.

We can map this result to the one obtained in the quantum metrology community [27-31] with a few simplifications. The quantum metrology result considers the effect of optical loss on the optimal phase estimation in Michelson or equivalent Mach-Zehnder interferometers. We can match this if we ignore the internal squeezing or focus at high frequencies where the internal optomechanical squeezing is weak, such that $\alpha=1 / 4$, and also ignore the SRC loss. The corresponding sensitivity limit from the arm-cavity loss and the external loss is reduced to

$$
S_{h h}^{\epsilon}=\frac{\hbar c^{2}}{4 L^{2} \omega_{0} P}\left(\epsilon_{\mathrm{arm}}+\frac{T_{\mathrm{src}}}{4} \epsilon_{\mathrm{ext}}\right) .
$$

The extra factor of $T_{\mathrm{src}} / 4$ in front of $\epsilon_{\mathrm{ext}}$ can be intuitively understood as the suppression of the loss effect from the signal recycling. Furthermore, the impact of the losses discussed here is regularly included in the numerical computation of quantum noise in GW detectors $[32,33]$. These numerical 


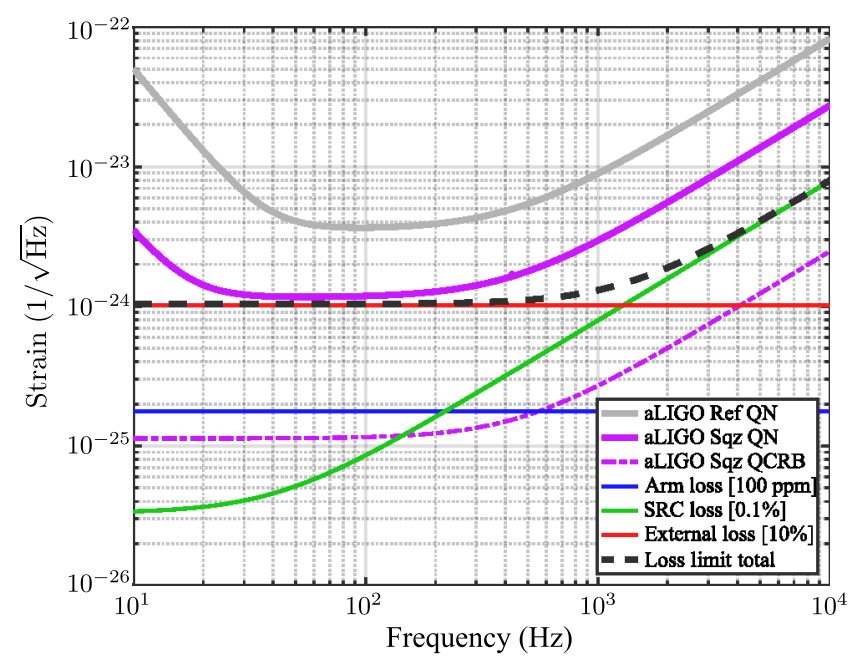

FIG. 2. This plot shows the quantum-noise (QN) sensitivity limits resulting from optical loss in an interferometer as given in Eq. (3) (blue, green, red, and dashed-black curves). For illustration, we assume a set of parameters similar to those of Advanced LIGO (aLIGO) and show the aLIGO QN for reference (upper light grey curve). To demonstrate the limited nature of the QCRB, the QN of aLIGO with a highly squeezed input state is shown (solid purple curve) along with the corresponding QCRB (dot-dashed purple curve). Notice how the QN of the highly squeezed interferometer is limited by optical losses, while the QCRB is significantly less constraining. Since the QCRB does not take into account losses, it can be made arbitrarily low by further increasing the squeezing of the input state, i.e., reducing the uncertainty in the phase quadrature, and thereby increasing $S_{P P}$ in Eq. (2) beyond the 30-dB level used for this example. Note also that the highly squeezed interferometer QN is above the loss limit (dashed black curve) at low frequencies $(\lesssim 20 \mathrm{~Hz})$ due to the fixed read-out quadrature and at high frequencies $(\gtrsim 400 \mathrm{~Hz})$ due to uncompensated dispersion.

computations clearly show that losses limit the detector sensitivity, but they do not provide insight into the configuration-independent limit these losses impose. Equation (3) goes beyond the case-by-case approach followed thus far to provide a general loss limit for GW detectors.

\section{DERIVATION}

Here, we show the details behind the main result, Eq. (3). We use the two-photon formalism developed by Caves and Schumaker [34]. In particular, we adopt the approach by Kimble et al. [9], which is based upon this formalism and especially tailored to the context of laser interferometric $\mathrm{GW}$ detectors. In this formalism, the optical field at different locations is fully described by its amplitude quadrature $\hat{a}_{1}$ and phase quadrature $\hat{a}_{2}$ :

$$
\hat{E}(t)=\hat{a}_{1}(t) \cos \omega_{0} t+\hat{a}_{2}(t) \sin \omega_{0} t .
$$

Moving into the frequency domain, $\hat{a}_{1,2}(\Omega)$ are labeled by the frequency $\Omega$, which is also called the sideband frequency and coincides with the GW signal frequency. Because the system is linear and time invariant, different frequency components are independent of each other. At each frequency, the effects of different optical elements on the quadratures can be quantified by $2 \times 2$ matrices, which act on the vector $\hat{\boldsymbol{a}}=\left(\hat{a}_{1}, \hat{a}_{2}\right)^{\prime}$. In particular, a passive element without external energy input is described by a rotation matrix:

$$
\mathbf{M}_{\mathrm{rot}}=\left[\begin{array}{cc}
\cos \Theta & -\sin \Theta \\
\sin \Theta & \cos \Theta
\end{array}\right] \text {. }
$$

For example, the Fabry-Perot filter cavity used for frequency-dependent squeezing (or read-out) leads to a frequency-dependent rotation angle $\Theta(\Omega)$ for the amplitude and phase quadratures. The angle can be tuned by changing the cavity bandwidth and detuning $[9,10]$. In general, there will be an additional phase factor $e^{i \Phi(\Omega)}$ in front of the rotation matrix for the full description of a passive element.

For a phase-sensitive active (squeezing) element [35], the corresponding matrix is

$$
\mathbf{M}_{\mathrm{sqz}}=\mathbf{M}_{\mathrm{rot}}(\theta)\left[\begin{array}{cc}
\mathrm{e}^{r} & 0 \\
0 & \mathrm{e}^{-r}
\end{array}\right] \mathbf{M}_{\mathrm{rot}}(-\theta) .
$$

where $r$ is the squeezing factor and $\theta$ is the squeezing angle: $r=1$ and $\theta=0$ correspond to about $9 \mathrm{~dB}$ of phase squeezing. In the most general cases, the relevant parameters are frequency dependent, namely, $\theta=\theta(\Omega)$ and $r=r(\Omega)$. For example, the internal ponderomotive (optomechanical) squeezing from the test-mass-light interaction inside the arm cavity can be described by the following matrix:

$$
\mathbf{M}_{\mathrm{opt}}=\left[\begin{array}{cc}
1 & 0 \\
-\kappa & 1
\end{array}\right]
$$

where $\kappa=16 P \omega_{0} /\left(M c^{2} \Omega^{2}\right)$. It can be decomposed into the rotation matrix $\mathbf{M}_{\mathrm{rot}}(\phi)$ followed by the general squeezing matrix $\mathbf{M}_{\mathrm{sqz}}(r, \theta)$, with

$$
\begin{gathered}
\phi=-\arctan (\kappa / 2), \quad \theta=\operatorname{arccot}(\kappa / 2) / 2, \\
r=-\operatorname{arcsinh}(\kappa / 2),
\end{gathered}
$$

as shown explicitly in Refs. $[9,38]$.

In Fig. 3(a), we show a simplified representation of the general scheme in Fig. 1 when only looking at the differential mode, which contains the GW signal, and the corresponding input and output fields at the differential (dark) port. We include the optical loss inside the arm cavity and the SRC and at the output. Depending on the configuration of the intracavity filters, the SRC loss can come from several lossy channels. Each introduces a frequency-dependent loss $\epsilon_{i}(\Omega)$. To derive the lower bound 


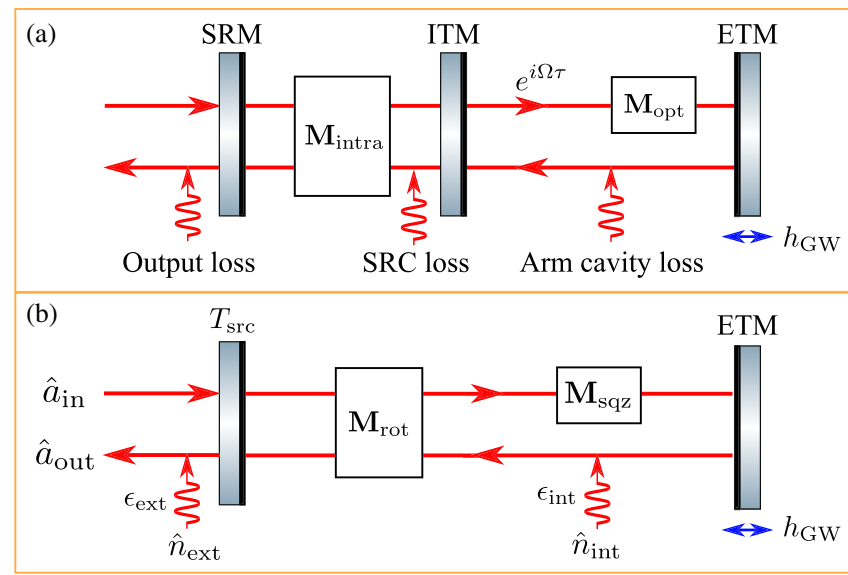

FIG. 3. (a) A simplified schematic of the general scheme shown in Fig. 1 when only focusing on the differential mode that contains the GW signal. The optical losses at different places are illustrated. (b) A further simplified diagram by mapping the SRC into an effective mirror, and optical losses into internal and external components. Here, $\mathbf{M}_{\text {rot }}$ accounts for a general rotation of the amplitude and phase quadratures; $\mathbf{M}_{\mathrm{sqz}}$ describes the general squeezing effect.

on the sensitivity without specifying the details of the intracavity filter configuration, we assume a single effective frequency-independent loss:

$$
\epsilon_{\mathrm{src}}=\min _{\Omega} \sum_{i} \epsilon_{i}(\Omega),
$$

which is the minimum of the total loss over the frequency.

Inside the arm cavity, the field not only experiences the ponderomotive squeezing mentioned earlier but also picks up a round-trip phase of $2 \Omega L / c$ through free propagation. The higher the frequency of the sideband, the more phase it picks up; this is sometimes referred to as "positive dispersion." Such a phase relation makes the high-frequency signal sidebands deviate from the perfect-resonance condition, which reduces their amplitude at the output. In the case of Advanced LIGO, it decreases the detector sensitivity at high frequencies and leads to a finite detector bandwidth around $1 \mathrm{kHz}$, as shown by the sensitivity curve in Fig. 2. With the nominal configuration, one can increase the bandwidth by tuning the properties of the SRC. However, this increase comes at the cost of decreasing the peak sensitivity. Such a trade-off between the bandwidth and peak sensitivity was first found by Mizuno [39] and is a direct consequence of the QCRB in Eq. (2).

Adding any passive element to the intracavity filter will also introduce a frequency-dependent phase, as mentioned after Eq. (6). This trade-off cannot be circumvented with passive elements, but since we are only considering the sensitivity bound resulting from optical losses, we assume that the intracavity filters may also contain active elements that produce the so-called white-light-cavity effect.
With active elements present, all signal sidebands may be resonantly enhanced [6-8]. In other words, these active elements produce the optimal phase $\phi_{\text {nd }}$ with a negative dispersion, which cancels both the propagation phase $2 \Omega L / c$ and the passive filter phase $\Phi$ around the frequency of interest, namely,

$$
\phi_{\mathrm{nd}}(\Omega)=-\Phi(\Omega)-2 \Omega L / c
$$

Any nonperfect cancelation will lead to a sensitivity that is worse than the loss-induced limit considered here.

With the assumption expressed in Eq. (9) and the condition given in Eq. (10), we can further simplify the general scheme into what is shown in Fig. 3(b): $\mathbf{M}_{\text {rot }}$ and $\mathbf{M}_{\text {sqz }}$ capture the effect of both the internal squeezing and the intracavity filters, which gives rise to $\mathbf{M}_{\text {intra }}$ (a general rotation and squeezing matrix). The optical losses $\epsilon_{\text {arm }}$ and $\epsilon_{\mathrm{src}}$ can then be combined to form a lower bound on the effective internal loss,

$$
\epsilon_{\mathrm{int}}=\epsilon_{\mathrm{arm}}+\frac{T_{\mathrm{itm}}}{4}\left(1+\frac{\Omega^{2}}{\gamma^{2}}\right) \epsilon_{\mathrm{src}} .
$$

The frequency-dependent factor in front of $\epsilon_{\text {src }}$ comes from the finite arm-cavity bandwidth. We have assumed frequencies that are lower than the free spectral range (FSR) $f_{\text {fsr }}=$ $c /(2 L)$ of the arm cavity. If this assumption is violated and the frequency is around other FSR, $\Omega$ must be replaced by $\Omega-2 n \pi f_{\text {fsr }}$, where $n$ is the index of the closest FSR.

Following the propagation of the optical field and using the continuity condition at different interfaces, we can derive the frequency-domain input-output relation for the scheme in Fig. 3(b):

$\hat{\boldsymbol{a}}_{\text {out }}=\mathbf{M}_{\mathrm{io}} \hat{\boldsymbol{a}}_{\mathrm{in}}+\sqrt{T_{\mathrm{src}} \epsilon_{\mathrm{int}}} \mathbf{M}_{\mathrm{c}} \hat{\boldsymbol{n}}_{\mathrm{int}}+\sqrt{\epsilon_{\mathrm{ext}}} \hat{\boldsymbol{n}}_{\mathrm{ext}}+\boldsymbol{v} h_{\mathrm{GW}}$,

which quantifies the noise and signal content in the output at each frequency. Here, $\hat{\boldsymbol{a}}$ and $\hat{\boldsymbol{n}}$ with different subscripts are vectors of the amplitude and phase quadratures of fields at different locations. We do not include the input loss explicitly, which can be accounted for by assuming some degradation on the input squeezing level if the squeezed light is used. The transfer matrix $\mathbf{M}_{\text {io }}$ between $\hat{\boldsymbol{a}}_{\text {in }}$ and $\hat{\boldsymbol{a}}_{\text {out }}$ is defined as

$$
\mathbf{M}_{\mathrm{io}} \equiv-\sqrt{R_{\mathrm{src}}} \mathbf{I}+T_{\mathrm{src}} \mathbf{M}_{\mathrm{c}} \mathbf{M}_{\mathrm{rot}} \mathbf{M}_{\mathrm{sqz}} \mathbf{M}_{\mathrm{rot}}
$$

in which $\mathbf{I}$ is the identity matrix, $R_{\mathrm{src}} \equiv 1-T_{\mathrm{src}}$, and the transfer matrix $\mathbf{M}_{\mathrm{c}}$ is defined as $\left[\mathbf{I}-\sqrt{R_{\mathrm{src}}} \mathbf{M}_{\mathrm{rot}} \mathbf{M}_{\mathrm{sqz}} \mathbf{M}_{\mathrm{rot}}\right]^{-1}$. The vector $\boldsymbol{v}$ describes the detector response to the $\mathrm{GW}$ signal: 


$$
\boldsymbol{v} \equiv \sqrt{T_{\mathrm{src}}} \mathbf{M}_{\mathrm{c}} \boldsymbol{v}_{0}
$$

with $v_{0}=(0, \beta)^{\prime}$ and $\beta=2 \sqrt{\omega_{0} L^{2} P /\left(\hbar c^{2}\right)}$.

Using the homodyne read-out, we can measure the general quadrature of the outgoing field $\hat{a}_{\text {out }}(\zeta)$, which is equal to $(\cos \zeta, \sin \zeta) \hat{\boldsymbol{a}}_{\text {out }}$. If we apply an output filter that optimizes $\zeta$ at all frequencies, we will obtain the best sensitivity with the minimum signal-referred noise spectral density:

$$
S_{h h}^{\min }=\frac{1}{\boldsymbol{v}^{\dagger} \boldsymbol{\Sigma}_{\text {tot }}^{-1} \boldsymbol{v}} \approx S_{h h}^{\mathrm{QCRB}}+S_{h h}^{\epsilon},
$$

where $\boldsymbol{\Sigma}_{\text {tot }} \equiv \mathbf{M}_{\text {io }} \mathbf{M}_{\text {io }}^{\dagger}+T_{\text {src }} \epsilon_{\text {int }} \mathbf{M}_{\mathrm{c}} \mathbf{M}_{\mathrm{c}}^{\dagger}+\epsilon_{\text {ext }} \mathbf{I}$ is the total covariance matrix, and the approximation is to the first order in $\epsilon_{\text {int }}$ and $\epsilon_{\text {ext }}$.

Since the interferometer under consideration is a linear Gaussian system, we can view the signal part of Eq. (14) as displacing the mean of the Gaussian state of the optical field. We can obtain the sensitivity bound by evaluating the quantum Fisher information for estimating the mean of a Gaussian state [17,40-43]. This approach gives exactly the same result shown above; i.e., Eq. (15) is also the QCRB in the presence of optical loss, and the optimal homodyne detection is the one that saturates it. We intentionally separate the first term and call it $S_{h h}^{\mathrm{QCRB}}$ to echo the result presented in Refs. [14,16], where the lossless case was considered.

The explicit form of $S_{h h}^{\mathrm{QCRB}}$ and $S_{h h}^{\epsilon}$, in terms of $r, T_{\mathrm{src}}, \Theta$, and $\theta$, is quite complicated. However, not all parameter regimes are relevant. To achieve a low QCRB or large power fluctuation, we require $T_{\text {src }} \ll 1$ to enhance the signal recycling. We also assume $\Theta \ll 1$ to focus on relevant frequencies that are within one free spectral range of the arm cavity. The internal squeezing needs to be of the same order of $T_{\text {src }}$ so that the round-trip gain of the amplitude quadrature is close to unity, which can then result in a significant level of power fluctuation. In such a parameter regime, we can make a Taylor expansion of both $S_{h h}^{\mathrm{QCRB}}$ and $S_{h h}^{\epsilon}$ with respect to these small parameters. Specifically, up to the leading order of $T_{\text {src }}, \Theta$, and $r$, we have

$S_{h h}^{\mathrm{QCRB}}=\frac{\hbar c^{2}\left(\delta^{2}-4 r^{2}\right)^{2} e^{-2 r_{\text {input }}}}{16 L^{2} \omega_{0} P T_{\text {src }}\left[\delta^{2}+4 r^{2}+4 \delta r \sin \left(\theta+\theta_{0}\right)\right]}$,

where $r_{\text {input }}$ is the squeezing factor of the input squeezed light, $\delta \equiv \sqrt{T_{\text {src }}^{2}+16 \Theta^{2}}$ and $\theta_{0} \equiv \cot ^{-1}\left(4 \Theta / T_{\text {src }}\right)$. This means that we can make the above QCRB vanish, i.e., achieving an unbounded sensitivity in the ideal lossless case, if $r=\delta / 2$. Under this condition, the resulting sensitivity limit due to the optical loss reads

$$
\min _{\theta} S_{h h}^{\epsilon}=\frac{\hbar c^{2}}{4 L^{2} \omega_{0} P}\left(\epsilon_{\mathrm{int}}+T_{\mathrm{src}} \epsilon_{\mathrm{ext}}\right)
$$

where the minimum is achieved by setting $\theta=\pi / 2+\theta_{0}$.

If, however, the internal squeezing is negligible with $r=0$, the QCRB cannot be made to be arbitrarily small given a finite input squeezing, and we have

$$
S_{h h}^{\mathrm{QCRB}}=\frac{\hbar c^{2} \delta^{2} e^{-2 r_{\text {input }}}}{16 T_{\mathrm{src}} L^{2} \omega_{0} P},
$$

which is simply the shot-noise-only sensitivity given a general $\Theta$. The corresponding loss-induced limit is

$$
\min _{\phi} S_{h h}^{\epsilon}=\frac{\hbar c^{2}}{4 L^{2} \omega_{0} P}\left(\epsilon_{\mathrm{int}}+\frac{T_{\mathrm{src}}}{4} \epsilon_{\mathrm{ext}}\right)
$$

where the minimum is attained when $\Theta=0$ (tuned). Equations (17) and (19) together give our main result shown in Eq. (3), when expanding out $\epsilon_{\text {int }}$ in terms of $\epsilon_{\text {arm }}$ and $\epsilon_{\text {src }}$.

It is worth pointing out that there is a factor of 4 difference in the dependence of $\epsilon_{\text {ext }}$ between Eqs. (17) and (19). It originates from a reduced signal response when trying to maximize the sensitivity using the internal squeezing:

$$
\frac{\left|\boldsymbol{q}_{\zeta_{\mathrm{op}}} \boldsymbol{v}(r=\delta / 2)\right|}{\left|\boldsymbol{q}_{\zeta} \boldsymbol{v}(r=0)\right|}=\left[\frac{1+\sin \left(\theta+\theta_{0}\right)}{4}\right]^{1 / 2} \leq \frac{1}{2},
$$

which shows that the signal response with internal squeezing is at least a factor of 2 smaller. Such a reduction of signal response was mentioned by Peano et al. [24] and recently investigated experimentally by Korobko et al. [25], when considering placing an internal frequency-independent squeezer inside an optical cavity. In the case of laser interferometric GW detectors, even without introducing an additional squeezer, as mentioned earlier, there is internal ponderomotive squeezing. The only difference is that the corresponding squeezing factor $r$ is highly frequency dependent due to the test mass response [cf. Eq. (8)], and the condition $r=\delta / 2$ can only be satisfied at a single frequency, given the nominal dual-recycled Michelson interferometer, which is also illustrated in Appendix A.

\section{CONCLUSIONS AND DISCUSSIONS}

We present a new fundamental limit to gravitationalwave detector sensitivity based on optical losses which lead to decoherence. While the quantum Cramér-Rao bound in Eq. (2) provides a fundamental limit to the sensitivity of gravitational-wave detectors in the ideal lossless case, the optical-loss-induced limit presented in Eq. (3) can be more stringent. Unlike the QCRB, the loss limit cannot be made irrelevant with high levels of external or internal squeezing. 
The implication of our study for future gravitational-wave detectors is that the minimization of optical losses in the interferometer arms and in the read-out must be a strong focus of research and development efforts.

There are three additional points that we would like to mention to broaden the scope and applicability of our result. First, while we have focused our discussion on Michelson-type interferometers, there are advanced QND configurations based on Sagnac interferometers [44,45]. Optical loss is also an important limiting factor for such quantum speed meters [46], and our result can be directly applied to the equivalent sloshing-cavity-based speedmeter scheme [47]. For Sagnac speed meters with additional intracavity and output filters, the term in Eq. (3) from the arm-cavity loss is still the same, but we need to introduce an additional factor of $\left(\omega_{s} / \Omega\right)^{2}$ at frequencies below $\omega_{s}$ (the characteristic frequency of the speed response) for the limit from the SRC loss and the external loss. This factor accounts for the difference between the position response and the speed response at low frequencies.

Second, our result also applies to any optomechanical sensors that can be put into the general scheme illustrated in Fig. 3(b). They share the same principle as the laser interferometric gravitational-wave detectors, even though they may operate in different parameter regimes and have different forms of ponderomotive squeezing $[22,48]$. The only issue occurs around the resonant frequency of the mechanical oscillator, where, if the optical loss is small, the loss limit may not be the dominant one and the zero-point fluctuation (ZFP) of the mechanical oscillator will impose a more stringent bound [36,37]. Moreover, the quantum noise of the light cannot be made lower than the mechanical zero-point fluctuation at the resonance using the homodyne detection [49-51]; the total quantum noise is actually twice (in power) the noise from zero-point fluctuations-an interesting consequence of the linear quantum measurement theory $[52,53]$.

Finally, as illustrated in Appendix B, the loss effect described here is analogous to thermal noise from the mechanical dissipation, which provides another fundamental limit to gravitational-wave detectors and other highprecision measurements. This not only highlights the fundamental role of optical losses in quantum metrology but also motivates future studies, which may eventually allow us to understand both the mechanical dissipation and the optical dissipation under a unified framework.

\section{ACKNOWLEDGMENTS}

We would like to thank Rana Adhikari, Lisa Barsotti, Yanbei Chen, Stefan Danilishin, Jan Harms, Farid Khalili, Mikhail Korobko, Yiqiu Ma, Denis Martynov, as well as members of the LSC AIC and QN groups for fruitful discussions. H. M. is supported by UK STFC Ernest Rutherford Fellowship (Grant No. ST/M005844/11).
M. E. acknowledges the support of the National Science Foundation and the LIGO Laboratory. LIGO was constructed by the California Institute of Technology and Massachusetts Institute of Technology with funding from the National Science Foundation and operates under Cooperative Agreement No. PHY-0757058.

\section{APPENDIX A: UNDERSTANDING THE OPTICAL SPRING FROM INTERNAL PONDEROMOTIVE SQUEEZING}

Here, we provide some insight into the optical spring effect in the laser interferometric GW detectors from the perspective of internal ponderomotive squeezing. Conventionally, the optical spring is understood in terms of a modification to the dynamics of the test mass through an optical feedback; the free mass is effectively turned into a harmonic oscillator that resonates at the optical spring frequency [23]. In this picture, the enhancement of the sensitivity is attributed to an increase of the response to the GW signal (viewed as a tidal force acting on the test mass). However, as we have seen from the discussion about the internal squeezing, if we choose the optimal read-out quadrature for maximizing the sensitivity [cf. Eq. (20)], the signal response is decreased by a factor of 2 compared with the case without using the internal squeezing. This result seems to be in contradiction with the interpretation of the optical spring effect as arising from the internal ponderomotive squeezing [16]. It can be clarified if we look at the noise amplitude and signal response that define the sensitivity separately.

In Fig. 4, we compare two cases: one with tuned SRC and the other with detuned SRC. The optical spring effect is present in the latter. At the optical spring frequency, the signal response is amplified compared to the tuned case without the optical spring, when we measure the output phase quadrature (the default quadrature that GW detectors measure). However, the corresponding noise amplitude is also high, leading to a suboptimal sensitivity. If instead the optimal quadrature is measured, we can achieve the best sensitivity at the optical spring frequency, which was first presented in Ref. [54]. The main contribution comes from a significant reduction of the noise amplitude due to the internal ponderomotive squeezing. The signal response for the optimal quadrature read-out is indeed reduced by a factor of 2 compared with the tuned case with phase quadrature measurement, agreeing with our statement earlier. Therefore, reaching the optimal sensitivity using the optical spring effect is mostly attributable to the squeezing rather than the signal enhancement. It is worth mentioning that we are considering the presence of a small amount of optical loss. If the output loss is very high and significantly degrades the generated squeezing, the optimal quadrature for maximizing the sensitivity can deviate from the one with a reduced signal response, and having signal enhancement can be preferable [55]. 


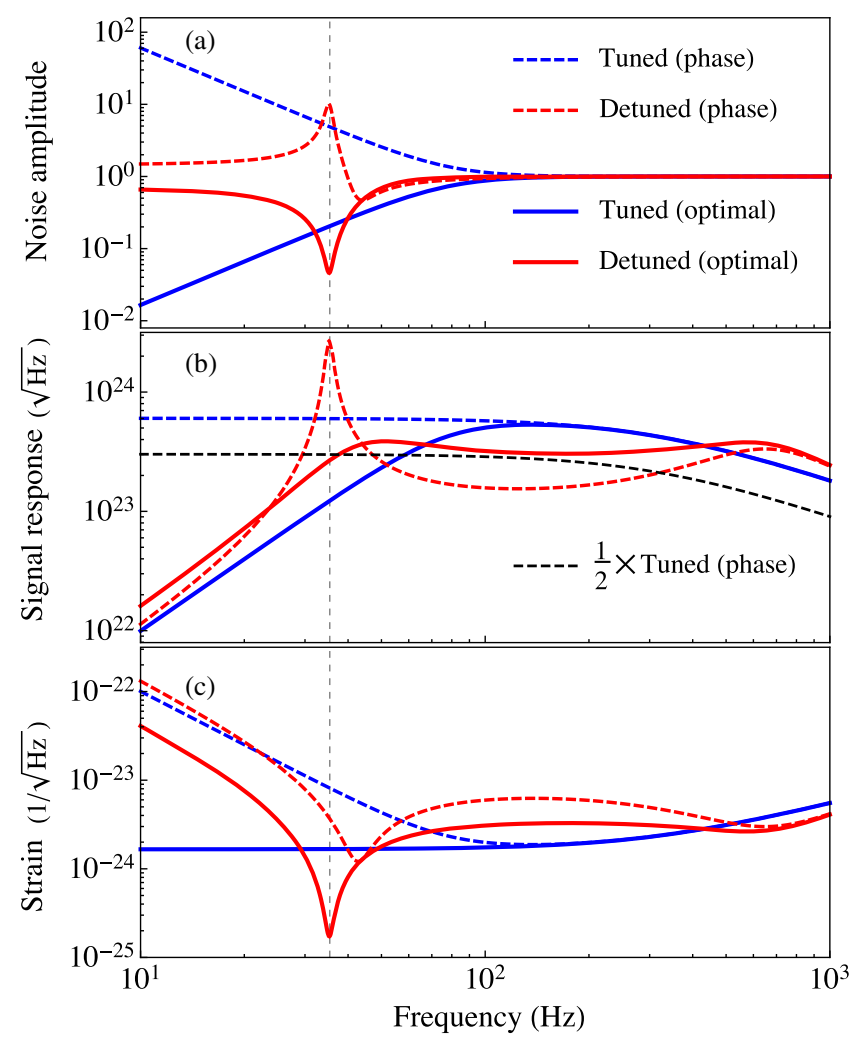

FIG. 4. (a) The noise amplitude, given the measurement of output phase quadrature (dashed line) and the optimal quadrature (solid line) in the two cases: tuned SRC (blue) and detuned SRC (red), respectively. (b) The corresponding signal response for these two cases. We also show the half of the signal response with a phase measurement in the tuned case as a reference (dashed black curve). (c) The sensitivity curve, which is a ratio of the noise amplitude in (a) and the signal response in (b). We assume the interferometer parameters are the same as the aLIGO design, but we choose the detector bandwidth to be $240 \mathrm{~Hz}$ and the detuning to be $600 \mathrm{~Hz}$ for illustration. The optical spring frequency is marked using the vertical grid line around $35 \mathrm{~Hz}$.

\section{APPENDIX B: OPTICAL FLUCTUATION- DISSIPATION THEOREM}

The loss-induced quantum limit can be viewed as arising from the optical dissipation, analogous to the thermal noise from the mechanical dissipation. Such an analogy, in some limiting cases, can be made exact by using the fluctuationdissipation theorem (FDT) [56]. In the GW community, the FDT has been widely applied for analyzing other classical fundamental limits to GW detectors, arising from the thermal noise in the suspension and test-mass mirrors [57-59]. The idea of applying FDT to the optical degrees of freedom (d.o.f.) was first envisioned by Smith and Levin [60]. The goal is to provide a unified treatment of the quantum noise and the classical thermal noise. However, the nonequilibrium nature of the quantum measurement process defies a straightforward generalization. In particular, we have a coherent laser field driving the system out of equilibrium, which induces internal ponderomotive squeezing. Interestingly, as we have seen from the previous analysis, the internal squeezing only changes the loss limit by a factor of 4 without changing the dependence to other parameters. Therefore, the equilibrium FDT applied to the case without the internal squeezing can provide some quantitative and useful insights into the loss limit, like what we are trying to show in the discussion below.

Since we are looking at the optical frequency, the external continuum field introduced by the optical loss, to a good approximation, can be treated as a zerotemperature heat bath. As a result, according to the FDT [56,61], any dynamical quantity $x$ of the system, which couples to the continuum field, satisfies

$$
S_{x x}(\omega)=2 \hbar \operatorname{Im}\left[\chi_{x x}(\omega)\right],
$$

where $\chi_{x x}$ is the susceptibility of $x$ and $\operatorname{Im}\left[\chi_{x x}\right]$ is its imaginary part, which quantifies the dissipation.

In our case, the system is the optical field at different locations, which couples to the heat bath due to the presence of the optical loss and also responds to the GW signal. Let us use the field inside the arm cavity and the associated arm-cavity loss to illustrate the basic idea. At frequencies below the free spectral range $c / 2 L$, the arm-cavity field can be modeled as a single mode or a simple damped harmonic oscillator: Its amplitude quadrature $\hat{A}_{1}$ and phase quadrature $\hat{A}_{2}$ satisfy the following equations of motion:

$$
\begin{aligned}
& \dot{\hat{A}}_{1}(t)+\gamma_{\epsilon} \hat{A}_{1}(t)=\omega_{\mathrm{cav}} \hat{A}_{2}(t)+\sqrt{2 \gamma_{\epsilon}} \hat{A}_{1}^{\mathrm{ext}}(t), \\
& \dot{\hat{A}}_{2}(t)+\gamma_{\epsilon} \hat{A}_{2}(t)=-\omega_{\text {cav }} \hat{A}_{1}(t)+\sqrt{2 \gamma_{\epsilon}} \hat{A}_{2}^{\mathrm{ext}}(t),
\end{aligned}
$$

where $\omega_{\text {cav }}$ is the cavity resonant frequency, $\gamma_{\epsilon}=$ $c \epsilon_{\text {arm }} /(4 L)$ is the damping rate due to the arm-cavity loss, and $\hat{A}^{\text {ext }}$ is the external continuum field. In the linearresponse theory $[16,23,56]$, the susceptibility is defined as

$$
\chi_{A B}\left(t-t^{\prime}\right) \equiv(i / \hbar)\left[\hat{A}(t), \hat{B}\left(t^{\prime}\right)\right] \Theta\left(t-t^{\prime}\right) .
$$

Using the commutator relation $\left[\hat{A}_{1}^{\mathrm{ext}}(t), \hat{A}_{2}^{\mathrm{ext}}\left(t^{\prime}\right)\right]=$ $i \hbar \delta\left(t-t^{\prime}\right)$, we can derive the relevant susceptibilities $\chi_{A_{2} A_{2}}$ and $\chi_{A_{2} A_{1}}$. In the frequency domain, they can be written as

$$
\begin{aligned}
& \chi_{A_{2} A_{2}}(\omega)=\frac{\omega_{\mathrm{cav}}}{\hbar\left[\left(\gamma_{\epsilon}-i \omega\right)^{2}+\omega_{\mathrm{cav}}^{2}\right]}, \\
& \chi_{A_{2} A_{1}}(\omega)=\frac{i \omega-\gamma_{\epsilon}}{\hbar\left[\left(\gamma_{\epsilon}-i \omega\right)^{2}+\omega_{\mathrm{cav}}^{2}\right]} .
\end{aligned}
$$

In the transverse-traceless (TT) gauge [62,63], a GW acts as a strain directly coupled to the cavity mode, of which the linearized interaction Hamiltonian is

$$
\hat{H}^{\mathrm{TT}}=-\hbar g \hat{A}_{1} L h_{\mathrm{GW}} .
$$


Here, we have defined $g \equiv 2 \sqrt{P \omega_{0} /(\hbar L c)}$, and $h_{\mathrm{GW}}$ is the GW strain. With the FDT in Eq. (B1) and the susceptibility in Eq. (B5), the sensitivity limit due to arm-cavity loss can be obtained by normalizing the fluctuation of the phase quadrature with respect to the signal response obtained from Eqs. (B6) and (B7), namely,

$$
S_{h h}^{\epsilon}(\omega)=\frac{S_{A_{2} A_{2}}(\omega)}{\hbar^{2} g^{2} L^{2}\left|\chi_{A_{2} A_{1}}(\omega)\right|^{2}}=\frac{2 \operatorname{Im}\left[\chi_{A_{2} A_{2}}(\omega)\right]}{\hbar g^{2} L^{2}\left|\chi_{A_{2} A_{1}}(\omega)\right|^{2}} .
$$

Switching to the sideband frequency $\Omega$ with the laser frequency $\omega_{0}$ as the reference, we obtain the first term in Eq. (3):

$$
S_{h h}^{\epsilon}(\Omega)=\frac{4 \gamma_{\epsilon} \omega \omega_{\mathrm{cav}}}{\left(\omega^{2}+\gamma_{\epsilon}^{2}\right) g^{2} L^{2}} \approx \frac{\hbar c^{2} \epsilon_{\mathrm{arm}}}{4 L^{2} \omega_{0} P},
$$

where we have used the fact that $\omega_{\text {cav }}$ is approximately equal to $\omega$ with $\omega=\Omega+\omega_{0} \approx \omega_{0}$, and $\omega_{0} \gg \gamma_{\epsilon}$.

The above result still holds even when the arm-cavity mode is coupled to additional d.o.f. This is because the ratio $\operatorname{Im}\left[\chi_{A_{2} A_{2}}\right] /\left|\chi_{A_{2} A_{1}}\right|^{2}$ is an invariant, as long as there is no dissipation in these additional d.o.f. and they are passive. To prove this, we can have the phase quadrature of the cavity mode coupled to some general coordinate $\hat{y}$ of one of these d.o.f. Using the linear-response theory, the coupling modifies the original susceptibilities $\chi_{A_{2} A_{2}}$ and $\chi_{A_{2} A_{1}}$ into

$\chi_{A_{2} A_{2}}^{\text {new }}=\frac{\chi_{A_{2} A_{2}}}{1-\chi_{A_{2} A_{2}} \chi_{y y}}, \quad \chi_{A_{2} A_{1}}^{\text {new }}=\frac{\chi_{A_{2} A_{1}}}{1-\chi_{A_{2} A_{2}} \chi_{y y}}$.

Since no dissipation is present in these d.o.f., i.e., $\operatorname{Im}\left[\chi_{y y}\right]=0$, we have

$\operatorname{Im}\left[\chi_{A_{2} A_{2}}^{\text {new }}\right]=\frac{\operatorname{Im}\left[\chi_{A_{2} A_{2}}\left(1-\chi_{A_{2} A_{2}}^{*} \chi_{y y}\right)\right]}{\left|1-\chi_{A_{2} A_{2}} \chi_{y y}\right|^{2}}=\frac{\operatorname{Im}\left[\chi_{A_{2} A_{2}}\right]}{\left|1-\chi_{A_{2} A_{2}} \chi_{y y}\right|^{2}}$.

This result implies $\operatorname{Im}\left[\chi_{A_{2} A_{2}}^{\text {new }}\right] /\left|\chi_{A_{2} A_{1}}^{\text {new }}\right|^{2}=\operatorname{Im}\left[\chi_{A_{2} A_{2}}\right] /\left|\chi_{A_{2} A_{1}}\right|^{2}$, and regardless of the type of intracavity filters introduced, $S_{h h}^{\epsilon}(\Omega)$ is given by Eq. (B9) for arm-cavity loss.

Similarly, we can derive the result for the SRC loss and the output loss by converting them into an effective armcavity loss. However, such a conversion can be made exact only when we ignore the internal squeezing or tweak the signal response by compensating the additional factor of 4 difference mentioned earlier.

[1] V. B. Braginskii, Classical and Quantum Restrictions on the Detection of Weak Disturbances of a Macroscopic Oscillator, JETP 26, 831 (1968).

[2] V. B. Braginsky and F. Ya. Khalilli, Quantum Measurement (Cambridge University, Cambridge, England, 1992).

[3] V. B. Braginsky, Yu. I. Vorontsov, and K. S. Thorne, Quantum Nondemolition Measurement, Science 209, 547 (1980).
[4] S. L. Danilishin and F. Ya. Khalili, Quantum Measurement Theory in Gravitational-Wave Detectors, Living Rev. Relativity 15, 5 (2012).

[5] H. Miao, H. Yang, R. X. Adhikari, and Y. Chen, Quantum Limits of Interferometer Topologies for Gravitational Radiation Detection, Classical Quantum Gravity 31, 165010 (2014).

[6] A. Wicht, K. Danzmann, M. Fleischhauer, M. Scully, G. Müller, and R. Rinkleff, White-Light Cavities, Atomic Phase Coherence, and Gravitational Wave Detectors, Opt. Commun. 134, 431 (1997).

[7] H. Miao, Y. Ma, C. Zhao, and Y. Chen, Enhancing the Bandwidth of Gravitational-Wave Detectors with Unstable Optomechanical Filters, Phys. Rev. Lett. 115, 211104 (2015).

[8] M. Zhou, Z. Zhou, and S. M. Shahriar, Quantum Noise Limits in White-Light-Cavity-Enhanced Gravitational Wave Detectors, Phys. Rev. D 92, 082002 (2015).

[9] H. J. Kimble, Y. Levin, A. B. Matsko, K. S. Thorne, and S.P. Vyatchanin, Conversion of Conventional Gravitational-Wave Interferometers into Quantum Nondemolition Interferometers by Modifying Their Input and/or Output Optics, Phys. Rev. D 65, 022002 (2001).

[10] E. Oelker, T. Isogai, J. Miller, M. Tse, L. Barsotti, N. Mavalvala, and M. Evans, Audio-Band FrequencyDependent Squeezing for Gravitational-Wave Detectors, Phys. Rev. Lett. 116, 041102 (2016).

[11] R. Schnabel, Squeezed States of Light and Their Applications in Laser Interferometers, Phys. Rep. 684, 1 (2017).

[12] C. W. Helstrom, Minimum Mean-Squared Error of Estimates in Quantum Statistics, Phys. Lett. A 25, 101 (1967).

[13] A. Holevo, Probabilistic and Statistical Aspects of Quantum Theory, 2nd ed. (Scuola Normale Superiore, Pisa, 2011).

[14] V. B. Braginsky, M. L. Gorodetsky, F. Ya. Khalili, and K. S. Thorne, Energetic Quantum Limit in Large-Scale Interferometers, AIP Conf. Proc. 523, 180 (2000).

[15] M. Tsang, H. M. Wiseman, and C. M. Caves, Fundamental Quantum Limit to Waveform Estimation, Phys. Rev. Lett. 106, 090401 (2011).

[16] H. Miao, R. X. Adhikari, Y. Ma, B. Pang, and Y. Chen, Towards the Fundamental Quantum Limit of Linear Measurements of Classical Signals, Phys. Rev. Lett. 119, 050801 (2017).

[17] D. Branford, H. Miao, and A. Datta, Fundamental Quantum Limits of Multicarrier Optomechanical Sensors, Phys. Rev. Lett. 121, 110505 (2018).

[18] R. Lawrence, M. Zucker, P. Fritschel, P. Marfuta, and D. Shoemaker, Adaptive Thermal Compensation of Test Masses in Advanced LIGO, Classical Quantum Gravity 19, 1803 (2002).

[19] E. Hirose, K. Kawabe, D. Sigg, R. X. Adhikari, and P. R. Saulson, Angular Instability Due to Radiation Pressure in the LIGO Gravitational-Wave Detector, Appl. Opt. 49, 3474 (2010).

[20] V. B. Braginsky, S. E. Strigin, and S. P. Vyatchanin, Parametric Oscillatory Instability in Fabry-Pérot Interferometer, Phys. Lett. A 287, 331 (2001).

[21] M. Evans, S. Gras, P. Fritschel, J. Miller, L. Barsotti, D. Martynov, A. Brooks, D. Coyne, R. Abbott, R. X. Adhikari et al., Observation of Parametric Instability in Advanced LIGO, Phys. Rev. Lett. 114, 161102 (2015). 
[22] M. Aspelmeyer, T. J. Kippenberg, and F. Marquardt, Cavity Optomechanics, Rev. Mod. Phys. 86, 1391 (2014).

[23] A. Buonanno and Y. Chen, Signal Recycled LaserInterferometer Gravitational-Wave Detectors as Optical Springs, Phys. Rev. D 65, 042001 (2002).

[24] V. Peano, H. G. L. Schwefel, C. Marquardt, and F. Marquardt, Intracavity Squeezing Can Enhance QuantumLimited Optomechanical Position Detection through Deamplification, Phys. Rev. Lett. 115, 243603 (2015).

[25] M. Korobko, L. Kleybolte, S. Ast, H. Miao, Y. Chen, and R. Schnabel, Beating the Standard Sensitivity-Bandwidth Limit of Cavity-Enhanced Interferometers with Internal Squeezed-Light Generation, Phys. Rev. Lett. 118, 143601 (2017).

[26] M. Evans, L. Barsotti, P. Kwee, J. Harms, and H. Miao, Realistic Filter Cavities for Advanced Gravitational Wave Detectors, Phys. Rev. D 88, 022002 (2013).

[27] U. Dorner, R. Demkowicz-Dobrzanski, B. J. Smith, J. S. Lundeen, W. Wasilewski, K. Banaszek, and I. A. Walmsley, Optimal Quantum Phase Estimation., Phys. Rev. Lett. 102, 040403 (2009).

[28] J. Kolodynski and R. Demkowicz-Dobrzanski, Phase Estimation without a priori Phase Knowledge in the Presence of Loss, Phys. Rev. A 82, 053804 (2010).

[29] S. Knysh, V. N. Smelyanskiy, and G. A. Durkin, Scaling Laws for Precision in Quantum Interferometry and the Bifurcation Landscape of the Optimal State, Phys. Rev. A 83, 021804 (2011).

[30] B. M. Escher, R. L. de Matos Filho, and L. Davidovich, General Framework for Estimating the Ultimate Precision Limit in Noisy Quantum-Enhanced Metrology, Nat. Phys. 7, 406 (2011).

[31] R. Demkowicz-Dobrzanski, J. Kolodynski, and M. Guta, The Elusive Heisenberg Limit in Quantum-Enhanced Metrology, Nat. Commun. 3, 1063 (2012).

[32] L. Barsotti, J. Harms, and R. Schnabel, Squeezed Vacuum States of Light for Gravitational Wave Detectors, Rep. Prog. Phys. 82, 016905 (2018).

[33] H. Miao, H. Yang, and D. Martynov, Towards the Design of Gravitational-Wave Detectors for Probing Neutron-Star Physics, Phys. Rev. D 98, 044044 (2018).

[34] C. M. Caves and B. L. Schumaker, New Formalism for Two-Photon Quantum Optics. I-Quadrature Phases and Squeezed States. II-Mathematical Foundation and Compact Notation, Phys. Rev. A 31, 3068 (1985).

[35] A phase-insensitive element will introduce an idler noisy channel $[36,37]$. The resulting sensitivity limit is, in general, larger than the phase-sensitive one unless we measure the idler channel.

[36] C. M. Caves, Quantum Limits on Noise in Linear Amplifiers, Phys. Rev. D 26, 1817 (1982).

[37] A. A. Clerk, M. H. Devoret, S. M. Girvin, F. Marquardt, and R. J. Schoelkopf, Introduction to Quantum Noise, Measurement and Amplification, Rev. Mod. Phys. 82, 1155 (2010).

[38] P. Kwee, J. Miller, T. Isogai, L. Barsotti, and M. Evans, Decoherence and Degradation of Squeezed States in Quantum Filter Cavities, Phys. Rev. D 90, 062006 (2014).
[39] J. Mizuno, Comparison of Optical Configurations for LaserInterferometric Gravitational-Wave Detectors, Ph.D. thesis, University of Hannover (1995).

[40] O. Pinel, J. Fade, D. Braun, P. Jian, N. Treps, and C. Fabre, Ultimate Sensitivity of Precision Measurements with Intense Gaussian Quantum Light: A Multimodal Approach, Phys. Rev. A 85, 010101(R) (2012).

[41] A. Monras, Phase Space Formalism for Quantum Estimation of Gaussian States, arXiv:1303.3682.

[42] Y. Gao and H. Lee, Bounds on Quantum MultipleParameter Estimation with Gaussian State, Eur. Phys. J. D 68, 347 (2014).

[43] D. Šafránek, A. R. Lee, and I. Fuentes, Quantum Parameter Estimation Using Multi-mode Gaussian States, New J. Phys. 17, 073016 (2015).

[44] Y. Chen, Sagnac Interferometer as a Speed-Meter-Type, Quantum-Nondemolition Gravitational-Wave Detector, Phys. Rev. D 67, 122004 (2003).

[45] C. Gräf et al., Design of a Speed Meter Interferometer Proof-of-Principle Experiment, Classical Quantum Gravity 31, 215009 (2014).

[46] S. L. Danilishin, C. Gräf, S. S. Leavey, J. Hennig, E. A. Houston, D. Pascucci, S. Steinlechner, J. Wright, and S. Hild, Quantum Noise of Non-ideal Sagnac Speed Meter Interferometer with Asymmetries, New J. Phys. 17, 043031 (2015).

[47] P. Purdue and Y. Chen, Practical Speed Meter Designs for Quantum Nondemolition Gravitational-Wave Interferometers, Phys. Rev. D 66, 122004 (2002).

[48] Y. Chen, Macroscopic Quantum Mechanics: Theory and Experimental Concepts of Optomechanics, J. Phys. B 46, 104001 (2013).

[49] L. F. Buchmann, S. Schreppler, J. Kohler, N. Spethmann, and D. M. Stamper-Kurn, Complex Squeezing and Force Measurement Beyond the Standard Quantum Limit, Phys. Rev. Lett. 117, 030801 (2016).

[50] N. S. Kampel, R. W. Peterson, R. Fischer, P.-L. Yu, K. Cicak, R. W. Simmonds, K. W. Lehnert, and C. A. Regal, Improving Broadband Displacement Detection with Quantum Correlations, Phys. Rev. X 7, 021008 (2017).

[51] D. Mason, J. Chen, M. Rossi, Y. Tsaturyan, and A. Schliesser, Continuous Force and Displacement Measurement Below the Standard Quantum Limit, arXiv:1809 .10629.

[52] V. B. Braginsky and F. Ya. Khalili, Quantum Measurement (Cambridge University, Cambridge, England, 1992).

[53] F. Ya. Khalili, H. Miao, H. Yang, A. H. Safavi-Naeini, O. Painter, and Y. Chen, Quantum Back-Action in Measurements of Zero-Point Mechanical Oscillations, Phys. Rev. A 86, 033840 (2012).

[54] J. Harms, Y. Chen, S. Chelkowski, A. Franzen, H. Vahlbruch, K. Danzmann, and R. Schnabel, Squeezed-Input, Optical-Spring, Signal-Recycled Gravitational-Wave Detectors, Phys. Rev. D 68, 042001 (2003).

[55] This point was raised by Mikhail Korobko in a private communication.

[56] R. Kubo, The Fluctuation-Dissipation Theorem, Rep. Prog. Phys. 29, 255 (1966).

[57] P. R. Saulson, Fundamentals of Interferometric Gravitational Wave Detectors (World Scientific, Singapore, 1994). 
[58] Y. Levin, Internal Thermal Noise in the LIGO Test Masses: A Direct Approach, Phys. Rev. D 57, 659 (1998).

[59] G. M. Harry, H. Armandula, E. Black, D. R. M. Crooks, G. Cagnoli, J. Hough, P. Murray, S. Reid, S. Rowan, P. Sneddon, M. M. Fejer, R. Route, and S. D. Penn, Thermal Noise from Optical Coatings in Gravitational Wave Detectors, Appl. Opt. 45, 1569 (2006).

[60] N. D. Smith, and Y. Levin, Private communication.
[61] H. B. Callen and T. A. Welton, Irreversibility and Generalized Noise, Phys. Rev. 83, 34 (1951).

[62] F. I. Cooperstock and V. Faraoni, Laser-Interferometric Detectors of Gravitational Waves, Classical Quantum Gravity 10, 1189 (1993).

[63] S. P. Tarabrin and A. A. Seleznyov, Optical Position Meters Analyzed in the Noninertial Reference Frames, Phys. Rev. D 78, 062001 (2008). 